\title{
Inatividade física habitual e fatores associados em população nordestina atendida pela Estratégia Saúde da Família
}

\author{
Habitual physical inactivity and associated factors \\ in a population from northeastern Brazil attended \\ by the Family Health Strategy program
}

1 Universidade do Estado da Bahia, Departamento de Educação-Campus XII. Linha de Estudo Pesquisa e Extensão em Atividade Física. Guanambi, BA. Brasil.

2 Universidade Federal de Santa Catarina,

Centro de Desportos, Programa de Pós-Graduação em Educação Física, Núcleo de Pesquisa em Atividade Física e Saúde. Florianópolis, SC. Brasil.

3 Bolsista CNPq.

4 Bolsista Capes.

5 Universidade Federal de Santa Catarina, Centro Tecnológico, Departamento de Informática e Estatística. Florianópolis, SC. Brasil.

Recebido em 03/09/08 Revisado em 02/12/08 Aprovado em 10/03/09
Resumo - O objetivo deste estudo foi verificar a prevalência do baixo índice de atividade física habitual (BIAFH) em adultos e identificar associação com indicadores demográficos, socioeconômicos e de saúde. Realizou-se um estudo transversal, de base populacional domiciliar, em adultos de ambos os sexos, na faixa etária de 20 a 69 anos, residentes em oito áreas de cobertura da Estratégia Saúde da Família (ESF), da cidade de Guanambi (BA), Brasil. A representação domiciliar ficou composta por 565 domicílios, para uma amostra final de 711 adultos (93,15\%), sendo 544 (76,5\%) do sexo feminino. Para determinação do BIAFH, utilizou-se o questionário de Baecke, estabelecendo os quartis (primeiro e segundo), como recomendado pelo próprio instrumento $(\leq 7,25)$. Para as associações, aplicaram-se análises descritivas e a regressão de Poisson para uma significância de $\mathrm{p} \leq 0,05$. A prevalência de BIAFH foi de 51,1\%. Após análise ajustada, o BIAFH apresentou-se associado apenas com o sexo, com chance maior para as mulheres $(\mathrm{RP}=1,72, \mathrm{IC}=1,27-2,33)$. A prevalência de BIAFH foi semelhante à encontrada na literatura. É necessário que estudos associem atividades físicas, tais como, atividades domésticas, no lazer, no esporte ou ocupação/trabalho, com outras variáveis, mostrando as limitações e possibilidades para elaboração de programas de intervenção de atividade física na população atendida pela ESF.

Palavras-chave: Atividade Motora, Adultos, Prevalência, Atenção Primária, Programa Saúde da Família.

Abstract - The objective of this study was to determine the prevalence of low habitual physical activity (LHPA) in adults and to identify associated demographic, socioeconomic and health indicators. A household/population-based cross-sectional study was conducted on male and female adults aged 20 to 69 years, living in eight areas covered by the Family Health Strategy (FHS) program of the town of Guanambi, Bahia, Brazil. A total of 565 domestic households were included, for a final sample of 711 (93.15\%) adults, including 544 (76.5\%) females. The questionnaire of Baecke et al. was used to assess LHPA, establishing the first and second quartiles as recommended by the instrument itself $(\leq 7.25)$. Associations were analyzed using descriptive analysis and Poisson regression at a level of significance of $p \leq .05$. The prevalence of LHPA was $51.1 \%$. After adjusted analysis, LHPA was only associated with gender, with women presenting a higher chance of LHPA $(R R=1.72, \mathrm{CI}=1.27-2.33)$. The prevalence of LHPA was similar to that reported in the literature. Studies evaluating the association between physical activity such as domestic activities, leisure, sports or occupation/work and other variables are necessary to demonstrate the limitations and possibilities of physical activity intervention programs in populations attended by the FHS.

Key words: Motor activity; Adults; Prevalence; Primary Health Care; Family Health Program. 


\section{INTRODUÇÃO}

A prática de atividade física regular é reconhecida como fator de proteção para diversas doenças ${ }^{1} \mathrm{e}$ tem representado uma importante estratégia para a saúde pública ${ }^{2}$. Além de combater o crescimento das doenças crônicas, como hipertensão, diabetes, síndrome metabólica e obesidade, diminuem o impacto econômico para o indivíduo, família e sociedade, com custos de tratamento e medicamentos.

Estudos de associação da inatividade física com outros indicadores de saúde vêm crescendo 3 e, paralelamente a este aumento, a identificação dos diversos subgrupos expostos ao sedentarismo colabora na elaboração de políticas de incentivo a prática da atividade física.

Considerando-se todos os domínios (lazer, ocupação, deslocamento e exercício), no período de 1994 a 2005, a prevalência de inatividade física em adultos no Brasil esteve entre 26,7\% e 96,7\% . Estudos de base populacional indicam que estes valores estão em torno de $45 \%$ na população com idade acima de 14 anos $^{6,7}$.

As diferenças entre as prevalências podem ser justificadas pela utilização de diferentes instrumentos de medidas para avaliação do nível de atividade física. Apesar do IPAQ (Questionário Internacional de Atividade Física) ser utilizado com maior freqüência para identificação do sedentaris$\mathrm{mo}^{8}$, neste estudo optou-se pelo questionário de Baecke et al. ${ }^{9}$, por ser um instrumento prático para avaliar a atividade física habitual, aliando rapidez na aplicabilidade e facilidade no entendimento e preenchimento das respostas, sendo recomendada sua aplicação em estudos epidemiológicos ${ }^{10}$. O Índice de Atividade Física Habitual (IAFH) é caracterizado pelos domínios do trabalho e/ou na escola, das atividades esportivas, dos programas de exercícios físicos, do lazer ativo e das atividades de ocupação do tempo livre.

Na região Nordeste do Brasil, foram encontrados poucos estudos sobre prevalência da inatividade física associada com outros indicadores de saúde ${ }^{11,12}$, e apenas um deles refere-se à inatividade física em adultos no domínio do lazer ${ }^{11}$.

Esta pesquisa, além de procurar preencher esta lacuna na região nordeste, tem como objetivo verificar a prevalência do baixo índice de atividade física habitual (BIAFH) e identificar associação com indicadores demográficos, socioeconômicos e de saúde em adultos residentes e atendidos nas áreas de abrangência da ESF, na cidade de Guanambi, Bahia, Brasil.

\section{PROCEDIMENTOS METODOLÓGICOS}

Realizou-se um estudo transversal, de base populacional domiciliar, em adultos de ambos os sexos, com faixa etária entre 20 e 69 anos, residentes em oito áreas de cobertura da ESF, existentes na cidade de Guanambi (BA), a saber: Alto Caiçara, Alvorada, Beija Flor, Brasília, Lagoinha, Monte Pascoal, Vila Nova e Vomita Mel. Segundo dados de 2008, do Departamento de Saúde Coletiva da Prefeitura Municipal de Guanambi, a cobertura da ESF chega a $68,3 \%$, sendo $58,86 \%$ do Programa Saúde da Família e 9,44\% de cobertura de Saúde Bucal.

Guanambi é um município de médio porte, com cerca de $1260 \mathrm{~km}^{2}$, localizado no centro sul baiano, aproximadamente a $780 \mathrm{~km}$ da capital (Salvador), tendo como base econômica a indústria a pecuária e a agricultura. A população residente é de aproximadamente 72 mil habitantes, com uma expectativa de vida de 66,85 anos. A principal causa de mortalidade em adultos está relacionada às complicações no aparelho circulatório $(27,3 \%)^{13}$. O índice de desenvolvimento humano (IDH) é de $0,70^{13}$.

\section{Amostra}

Para o cálculo da amostra, tomou-se como base a população finita de 38.567 adultos, residentes em Guanambi/BA, com idade entre 20 e 69 anos $^{13}$, para uma estimativa de prevalência de inatividade física de $45 \%{ }^{14}$ e de erro amostral de três pontos percentuais, com nível de significância de 95\%. Após o cálculo, o número de domicílios a serem visitados seria de 671. Porém, em virtude das eventuais perdas e/ou recusas, foi adicionado $15 \%$ no número de domicílios, aproximando a um valor múltiplo de oito (número de áreas), sendo a representação final de 776 domicílios a serem visitados. Para distribuição por área, adotou-se como critério a quantidade de domicílios iguais para cada uma das oito áreas, correspondendo a 97 domicílios.

Depois de identificada a área de abrangência da regional de saúde, foram sorteados o setor, a rua e o lado de entrada de entrevistadores para início da coleta. Os domicílios foram escolhidos conforme os procedimentos apresentados pela pesquisa do INCA $/$ Brasil $^{15}$, com o critério de alternância, casa sim, casa não, sendo entrevistados todos os adultos encontrados em domicílio, em única visita.

Considerando as recusas e perdas (domiciliar e individual) a representação domiciliar ficou composta por 565 domicílios, distribuídos pelas oito áreas da ESF pesquisadas, para uma amostra final de 711 (93,15\%) adultos, com idade entre 20 e 69 anos 
(Figura 1), sendo 167 (23,5\%) do sexo masculino e $544(76,5 \%)$ do sexo feminino.

O número elevado de domicílios vazios (17,01\%) pode ser justificado pela sazonalidade (período de chuva), quando inicia o período de preparação do solo e plantação dos principais cultivos da região (algodão, milho, feijão e mandioca), tendo os adultos que se deslocar para áreas rurais mais distantes da cidade.

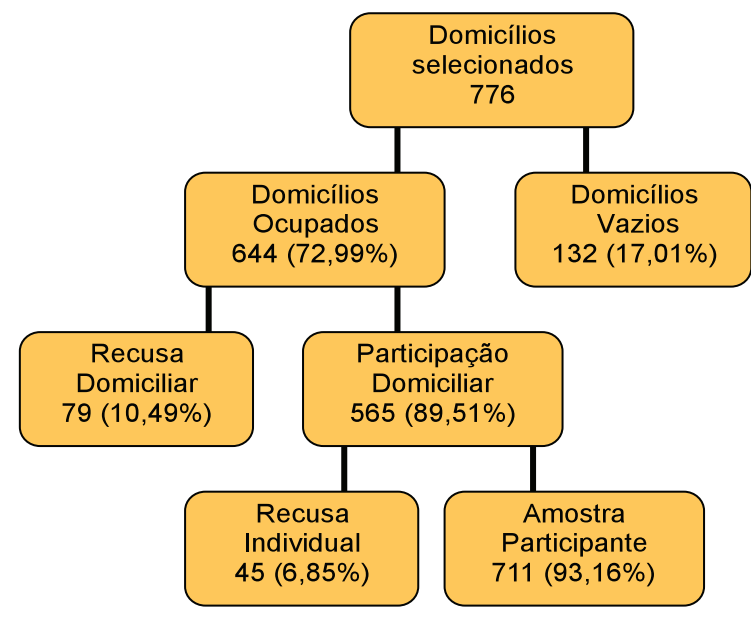

Figura 1. Representação domiciliar e individual da seleção da amostra nas oito áreas de cobertura da ESF, Guanambi, Bahia, Brasil (2007).

No presente estudo, foram consideradas as seguintes variáveis: como dependente, o Baixo Índice de Atividade Física Habitual (BIAFH) e como independentes sexo, faixa etária em anos completos (20-29; 30-39; 40-49; 50-59; 60 ou mais), massa corporal $(\mathrm{kg})$ e estatura (metros), estado civil (casado; solteiro e outros), escolaridade (analfabeto/fundamental incompleto; fundamental completo/médio incompleto; médio completo/superior incompleto; superior completo); situação ocupacional (trabalho; desempregado; dona (o) de casa; aposentado; outros), nível socioeconômico ${ }^{16}$ (alto: classes A e B; médio: classe $\mathrm{C}$ e baixo: classes $\mathrm{De} E$ ), percepção de saúde (positiva e negativa), tabagismo (fumante; exfumante; nunca fumou) e uso de bebidas alcoólicas (consumo frequente; consumo não freqüente);

\section{Coleta de dados}

As visitas aos domicílios aconteceram de segunda a sábado, em diferentes horários, quando se aplicou um questionário estruturado, em forma de entrevista, com duração de 10 a 20 minutos, em local reservado da residência.

A coleta de dados ocorreu de março a maio de 2007, com a participação de 20 entrevistadores, de ambos os sexos, estudantes do curso de Educação Física da Universidade do Estado da Bahia, que trabalharam em duplas (casal). Para aplicação do questionário estruturado (contendo 42 perguntas) e para padronização de medidas antropométricas (massa corporal e estatura), os entrevistadores foram submetidos a treinamento de sete dias, com aplicação de estudo piloto.

Para a verificação do Índice de Atividade Física Habitual, utilizou-se o questionário de Baecke et al. ${ }^{9}$, constituído por três partes distintas e composto por 16 questões com respostas indicadas em escala Likert de 5 pontos. As questões são direcionadas aos últimos 12 meses e associadas ao deslocamento/ locomoção para o trabalho e/ou na escola, às atividades esportivas, aos programas de exercícios físicos, ao lazer ativo e às atividades de ocupação do tempo livre. Para este estudo, o questionário de Baecke et al. foi devidamente pré-testado, obtendose um índice de concordância relativa de 77,86\%, para uma avaliação do índice de concordância de Kappa, "boa" ( $\mathrm{R}=0,73)$.

O BIAFH foi estabelecido de acordo com o primeiro e segundo quartis do índice de Baecke ${ }^{9}$ $(\leq 7,25)$, conforme critério recomendado pelos autores do instrumento. A classificação do BIAFH se referiu aos indivíduos que apresentavam os menores escores na soma das atividades de trabalho, exercício e no tempo livre.

A massa corporal foi aferida por meio da utilização da balança digital de bioimpedância Plenna (modelo Línea II); para aferição da estatura foi utilizada uma fita métrica em centímetros da marca Cardiomed. A partir das medidas antropométricas foi calculado o Índice de Massa Corporal (IMC), segundo as categorias da Organização Mundial de Saúde - $\mathrm{WHO}^{14}(\leq 18,4 ; 18,5$ - 24,9; 25,0 - 29,9; $\geq$ $30,0)$, que representa a razão da massa corporal pela estatura (em metros) elevada ao quadrado. As medidas foram realizadas em domicílio por um mesmo avaliador treinado utilizando-se dos procedimentos descrito por Alvarez e Pavan ${ }^{17}$.

O estudo foi desenvolvido de forma a proteger a privacidade dos indivíduos, garantindo a participação anônima e voluntária, com utilização do Termo de Consentimento Livre e Esclarecido, sendo seguidos, também, os critérios definidos pelo Conselho Nacional de Ética em Pesquisa (CONEP).

\section{Procedimentos de análise}

As variáveis contínuas (idade, massa corporal, estatura e índice de massa corporal - IMC) foram comparadas por meio do teste $\mathrm{t}$ de Student, para amostras independentes; para os percentuais das variáveis categóricas aplicou-se o teste do Qui quadrado $(\mathrm{p}<0,05)$. 
Para análise das variáveis associadas às prevalências do BIAFH, foram utilizadas a análise descritiva (distribuição de freqüências simples e percentuais) e regressão de Poisson, utilizando como medida de efeito a razão de prevalência (RP), bruta e ajustada, bem como seus intervalos de confiança (95\%), considerando os níveis de hierarquia. As variáveis pertencentes a cada nível de hierarquia são apresentadas na Tabela 3 .

Para a permanência das variáveis independentes no modelo final, foi utilizado o método de Stepwise.
Todas as variáveis que apresentaram $\mathrm{p}>0,20$ foram excluídas do modelo de regressão de Poisson ${ }^{18}$, permanecendo no modelo caso continuassem significantes $(\mathrm{p}<0,05)$ e/ou ajustassem o modelo. Os resultados foram analisados no programa estatístico Stata, versão 9.0 (Stata Corp, College Station, Estados Unidos).

\section{RESULTADOS}

Na Tabela 1 estão as características demográficas, comportamentais e de saúde da amostra (valores

Tabela 1. Descrição das características demográficas, comportamentais e de saúde (valores percentuais) dos adultos pertencentes às áreas de abrangência da ESF de Guanambi (BA), Brasil, 2007.

\begin{tabular}{|c|c|c|c|c|c|c|c|}
\hline \multirow[t]{2}{*}{ Caracterização } & \multicolumn{2}{|c|}{ masculino } & \multicolumn{2}{|c|}{ feminino } & \multicolumn{2}{|c|}{ Total } & \multirow[b]{2}{*}{ Valor de $p$} \\
\hline & $\mathrm{n}$ & $\%$ & $\mathrm{n}$ & $\%$ & $\mathrm{n}$ & $\%$ & \\
\hline \multicolumn{8}{|l|}{ Estado civil } \\
\hline Casado & 82 & 49,1 & 282 & 51,8 & 364 & 51,2 & \multirow[t]{2}{*}{0,80} \\
\hline Outros & 20 & 12,0 & 65 & 11,9 & 85 & 12,0 & \\
\hline 30 a 39 anos & 38 & 22,8 & 137 & 25,2 & 175 & 24,6 & \multirow{4}{*}{0,63} \\
\hline 40 a 49 anos & 28 & 16,8 & 101 & 18,6 & 129 & 18,1 & \\
\hline 50 a 59 anos & 27 & 16,2 & 66 & 12,1 & 93 & 13,1 & \\
\hline 60 anos ou mais & 12 & 7,2 & 47 & 8,6 & 59 & 8,3 & \\
\hline Médio completo/superior incompleto & 38 & 22,8 & 172 & 31,6 & 210 & 29,5 & \multirow{2}{*}{$0,04^{*}$} \\
\hline Superior completo & 03 & 1,8 & 15 & 2,8 & 18 & 2,5 & \\
\hline \multicolumn{8}{|l|}{ Nível Socioeconômico } \\
\hline Alta (classe A e B) & 10 & 6,0 & 27 & 5,0 & 37 & 5,2 & \multirow{3}{*}{0,84} \\
\hline Média (classe C) & 55 & 32,9 & 175 & 32,2 & 230 & 32,3 & \\
\hline Baixa (classe D e E) & 102 & 61,1 & 342 & 62,9 & 444 & 62,4 & \\
\hline \multicolumn{8}{|l|}{ Situação ocupacional } \\
\hline Trabalha & 104 & 62,3 & 164 & 30,1 & 268 & 37,7 & \multirow[b]{3}{*}{$<0,01^{*}$} \\
\hline Desempregado & 44 & 26,3 & 99 & 18,2 & 143 & 20,1 & \\
\hline Aposentado & 12 & 7,2 & 22 & 4,0 & 34 & 4,8 & \\
\hline \multicolumn{8}{|l|}{ Consumo de bebidas alcoólicas } \\
\hline Consumo frequente & 82 & 49,1 & 84 & 15,4 & 166 & 23,3 & \multirow{2}{*}{$<0,01^{*}$} \\
\hline Consumo não frequente & 85 & 50,9 & 460 & 84,6 & 545 & 76,7 & \\
\hline \multicolumn{8}{|l|}{ Percepção de saúde } \\
\hline Positiva & 105 & 62,9 & 328 & 60,3 & 433 & 60,9 & \multirow{2}{*}{0,30} \\
\hline Negativa & 62 & 37,1 & 216 & 39,7 & 278 & 39,1 & \\
\hline \multicolumn{8}{|l|}{ IMC } \\
\hline Baixo peso $(\leq 18,4)$ & 15 & 9,4 & 39 & 7,3 & 54 & 7,8 & \multirow{4}{*}{0,42} \\
\hline Faixa recomendável $(18,5-24,9)$ & 81 & 50,6 & 267 & 50,0 & 348 & 50,1 & \\
\hline Sobrepeso $(25,0-29,9)$ & 47 & 29,4 & 146 & 27,3 & 193 & 27,8 & \\
\hline Obesidade $(\geq 30,0)$ & 17 & 10,6 & 82 & 15,4 & 99 & 14,3 & \\
\hline
\end{tabular}

* Valor significativo $(\mathrm{p}<0,05)$. 
percentuais). A amostra foi representada por mulheres (76,5\%), indivíduos casados $(51,2 \%)$, na faixa etária entre 20 e 29 anos (35,9\%), por analfabetos ou com escolaridade no nível fundamental incompleto (48,2\%), de nível socioeconômico baixo $(62,4 \%)$, trabalhadores $(37,7 \%)$, não fumantes $(71,0 \%)$, com consumo não frequente de bebidas alcoólicas
(76,7\%), com percepção positiva de saúde $(60,9 \%)$ e com IMC na faixa recomendável (50,1\%).

Para a relação do sexo e as variáveis investigadas, encontraram-se diferenças significativas para a escolaridade $(p<0,04)$, a situação ocupacional $(\mathrm{p}<0,01)$, o uso do fumo $(\mathrm{p}<0,02)$ e o consumo de bebidas alcoólicas $(\mathrm{p}<0,01)$ (Tabela 1).

Tabela 2. Análise de regressão de Poisson do Baixo Índice de Atividade Física Habitual (BIAFH) com as características demográficas (nível 1), comportamentais (nível 2) e de saúde (nível 3) dos adultos pertencentes às áreas de abrangência da ESF de Guanambi (BA), Brasil, 2007.

\begin{tabular}{|c|c|c|c|c|c|}
\hline \multirow{2}{*}{ Nível } & \multirow{2}{*}{ Variáveis } & \multicolumn{2}{|c|}{ Prevalência BIAFH } & \multirow{2}{*}{$\begin{array}{c}\mathrm{RP}(\mathrm{IC}=95 \%) \\
\text { Regressão Poisson } \\
\text { bruta }\end{array}$} & \multirow{2}{*}{$\begin{array}{c}\mathrm{RP}(\mathrm{IC}=95 \%) \\
\text { Regressão Poisson } \\
\text { ajustada }\end{array}$} \\
\hline & & $\%$ & $\mathrm{n}$ & & \\
\hline \multirow[t]{3}{*}{1} & Sexo & & & $\mathrm{p} \leq 0,001^{*}$ & $\mathrm{p} \leq 0,01^{*}$ \\
\hline & Masculino & 29,9 & 50 & 1,00 & 1,00 \\
\hline & Feminino & 57,7 & 314 & $1,93(1,43$ a 2,60$)$ & $1,72(1,27$ a 2,33$)$ \\
\hline \multirow[t]{4}{*}{1} & Estado civil & & & $p=0,339$ & Excluído** \\
\hline & Solteiro & 46,6 & 122 & 1,00 & - \\
\hline & Casado & 54,9 & 200 & $1,18(0,94$ a 1,48$)$ & - \\
\hline & Outros & 49,4 & 42 & $1,06(0,75$ a 1,51$)$ & - \\
\hline \multirow[t]{6}{*}{1} & Faixa Etária & & & $p=0,607$ & Excluído** \\
\hline & 20 a 29 anos & 51,0 & 130 & 1,00 & - \\
\hline & 30 a 39 anos & 52,0 & 91 & $1,02(0,78$ a 1,33$)$ & - \\
\hline & 40 a 49 anos & 48,8 & 63 & $0,96(0,71$ a 1,29$)$ & - \\
\hline & 50 a 59 anos & 45,2 & 42 & $0,88(0,62$ a 1,25$)$ & - \\
\hline & 60 ou mais & 64,4 & 38 & $1,26(0,88$ a 1,81$)$ & - \\
\hline \multirow[t]{5}{*}{1} & Escolaridade & & & $p=0,621$ & Excluído** \\
\hline & Fundamental incompleto & 49,6 & 170 & 1,00 & \\
\hline & Fund completo/médio incompleto & 47,9 & 67 & $0,97(0,73$ a 1,28$)$ & \\
\hline & Médio completo/superior incompleto & 54,8 & 115 & $1,10(0,87$ a 1,40$)$ & \\
\hline & Superior completo & 66,7 & 12 & $1,34(0,75$ a 2,41$)$ & \\
\hline \multirow[t]{4}{*}{1} & Nível Sócioeconômico² & & & $P=0,675$ & Excluído** \\
\hline & Classe alta (A e B) & 59,5 & 22 & $1,14(0,74$ a 1,77$)$ & - \\
\hline & Classe média $(\mathrm{C})$ & 48,7 & 112 & $0,94(0,75$ a 1,17$)$ & - \\
\hline & Classe baixa (D e E) & 51,8 & 230 & 1,00 & - \\
\hline \multirow[t]{6}{*}{2} & Situação ocupacional & & & $\mathrm{P}=0,045^{*}$ & Excluído** \\
\hline & Trabalha & 41,0 & 110 & 1,00 & - \\
\hline & Desempregado & 54,5 & 78 & $1,32(0,99$ a 1,77$)$ & - \\
\hline & Dona de casa & 60,1 & 143 & $1,46(1,14$ a 1,88$)$ & - \\
\hline & Aposentado & 55,9 & 19 & $1,36(0,84$ a 2,21$)$ & - \\
\hline & Outros & 50,0 & 14 & $1,22(0,70$ a 2,12$)$ & - \\
\hline \multirow[t]{4}{*}{2} & Uso do fumo & & & $\mathrm{P}=0,174$ & $p=0,18$ \\
\hline & Nunca fumou & 53,1 & 268 & 1,00 & 1,00 \\
\hline & Ex-fumante & 55,4 & 46 & $1,04(0,76$ a 1,43$)$ & $1,05(0,77$ a 1,45$)$ \\
\hline & Fumante & 40,7 & 50 & $0,77(0,56$ a 1,04$)$ & $0,77(0,57$ a 1,05$)$ \\
\hline \multirow[t]{3}{*}{2} & Consumo de Bebidas Alcoólicas & & & $\mathrm{P}=0,015^{*}$ & Excluído** \\
\hline & Consumo freqüente & 39,8 & 66 & 1,00 & - \\
\hline & Consumo Não freqüente & 54,7 & 298 & $1,38(1,05$ a 1,80$)$ & - \\
\hline \multirow[t]{3}{*}{3} & Percepção da Saúde & & & $P=0,972$ & Excluído** \\
\hline & Positiva & 51,3 & 222 & 1,00 & - \\
\hline & Negativa & 51,1 & 142 & $0,99(0,81$ a 1,23$)$ & - \\
\hline \multirow[t]{5}{*}{3} & Índice de Massa Corporal (IMC) & & & $P=0,419$ & Excluído** \\
\hline & Baixo Peso $(\leq 18,4)$ & 51,9 & 28 & $0,97(0,65$ a 1,44$)$ & - \\
\hline & Faixa recomendável $(18,5-24,9)$ & 53,4 & 186 & 1,00 & - \\
\hline & Sobrepeso $(25,0-29,9)$ & 44,6 & 86 & $0,83(0,64$ a 1,07$)$ & - \\
\hline & Obesidade $(\geq 30,0)$ & 57,6 & 57 & $1,08(0,80$ a 1,45$)$ & - \\
\hline
\end{tabular}

*Diferença estatisticamente significativa. ** Excluído do modelo final $(p>0,20)$ 
O baixo índice de atividade física habitual (BIAFH) foi observado em 51,1\% ( $\mathrm{n}=364)$ do total da amostra participante.

$\mathrm{Na}$ Tabela 2, podem ser visualizadas as prevalências de BIAFH na amostra estudada (50 homens e 314 mulheres) em cada um dos níveis hierárquicos das variáveis (demográficas, comportamentais e de saúde), utilizando a análise bruta e ajustada pela regressão de Poisson. Na análise bruta, as mulheres tiveram quase duas vezes mais chance (93\%) de apresentar o BIAFH do que os homens. Na situação ocupacional, quando comparado aos que trabalhavam, o BIAFH foi $46 \%$ maior nos adultos que exerciam a atividade de "dona (o) de casa”. Após análise ajustada, as mulheres permaneceram apresentando associação estatisticamente significativa com o BIAFH. No modelo bruto, para a associação das prevalências do BIAFH com as variáveis comportamentais relacionadas à saúde, observou-se que para o consumo de bebidas alcoólicas, a probabilidade de apresentar BIAFH foi 38,0\% maior para os adultos que faziam uso não frequente; porém, essa variável deixou de ser significativa após ajuste para os níveis do modelo hierárquico.

\section{DISCUSSÃO}

Como parte de uma intervenção de atividade física para a Estratégia Saúde da Família (ESF), esta pesquisa teve como objetivo verificar a prevalência do baixo índice de atividade física habitual (BIAFH) e identificar associação com indicadores demográficos, socioeconômicos e de saúde em adultos residentes e atendidos nas áreas de abrangência da ESF, na cidade de Guanambi, Bahia, Brasil.

A prevalência do BIAFH observada neste estudo $(51,1 \%)$ indica que grande parcela da população investigada não atingi as recomendações quanto à prática de atividade física? ${ }^{7}$. A prevalência de inatividade física em adultos catarinenses apresenta-se entre 41,1\%6 a $57,4 \%{ }^{19}$. Quando considerada apenas a atividade física realizada no tempo de lazer, esta prevalência varia de $21,4 \%$ (pouco ativos) $^{20}$ a 89,7\% (pouco ativos) ${ }^{21}$.

$\mathrm{Na}$ amostra de Guanambi, o BIAFH apresentou associação com o sexo, sendo que as mulheres apresentaram maior probabilidade para um comportamento inativo ou com baixa atividade física. Diferença da inatividade física entre os sexos não foi observada em outras pesquisas, porém alguns estudos têm mostrado que as mulheres são mais inativas quando comparadas aos homens $s^{6,11,19}$.

A associação entre a prevalência do BIAFH e a situação ocupacional foi observada apenas na aná- lise bruta, sendo que as donas de casa apresentaram uma chance maior (significativa) de apresentar um comportamento inativo. No estudo de Baretta et al. ${ }^{19}$, a inatividade física apresentou-se positivamente associada a indivíduos que não trabalhavam ou não eram aposentados.

O consumo não frequente de bebidas alcoólicas foi associado com o BIAFH somente no modelo bruto. Dias-da-Costa et al. ${ }^{21}$ não encontraram diferença significativa do consumo de álcool com a inatividade física, no entanto, os autores observaram que a prevalência de inatividade física aumentou conforme a diminuição do consumo de bebidas alcoólicas, concordando com os resultados da presente pesquisa.

As outras variáveis analisadas neste estudo não mostraram associação com o BIAFH, sendo possível, portanto, apenas a observação das categorias das variáveis que apresentaram as maiores prevalências de baixo índice de atividade física.

Pesquisas têm mostrado maiores prevalências de inatividade física em indivíduos com um companheiro/casados ${ }^{6,20,21}$, nas pessoas com idade mais avançada $^{6,19,21}$, ex-fumantes ${ }^{6}$ e obesos ${ }^{19}$, o que vem a corroborar os achados do presente estudo. Prevalências maiores de inatividade física têm sido encontradas em pessoas com nível educacional mais baixo ${ }^{11,20}$ e de menor nível socioeconômico ${ }^{7,11,20,21}$.

Possivelmente, as pessoas de nível socioeconômico alto teriam melhores condições de acesso a programas de atividade física, facilitando um comportamento mais ativo. Ressalta-se, porém, que apenas 5,2\% da amostra deste estudo foi classificada no "nível socioeconômico alto", o que pode ter interferido na interpretação desses resultados, mostrando maior prevalência do BIAFH para esse grupo.

As prevalências de BIAFH em relação à percepção do estado de saúde positiva ou negativa foram quase idênticas (51,3 e 51,1\%). Alguns estudos $^{6,19}$ encontraram a percepção negativa de saúde mais frequente nos grupos fisicamente inativos.

O percentual de indivíduos com excesso de peso $(27,8 \%)$ e obesidade $(14,3 \%)$ encontrado neste estudo é semelhante ao observado no estudo de Coqueiro $^{22}$, em uma população de adultos atendidos pela ESF na cidade de Jequié (BA).

$\mathrm{O}$ alto percentual $(76,5 \%)$ de mulheres participantes da entrevista pode ser considerado um viés na escolha da amostra, já que os homens não tiveram a mesma oportunidade de participar do estudo. Isto pode ter ocorrido em virtude do horário das visitas domiciliares (horário comercial), com probabilidade maior das mulheres estarem em casa 
no momento da coleta. Isto também pode explicar o percentual de participantes "desempregados" (20,1\%) e "donas (os) de casa" (33,5\%).

Em virtude dos órgãos municipais não disponibilizarem o número de pessoas por área de cobertura das Unidades das Estratégias de Saúde da Família (UESF), adotou-se a distribuição proporcional da amostra para cada uma das oito áreas, evitando a extrapolação dos resultados para a população residente nas áreas de cobertura das ESF do município.

Apesar da boa reprodutibilidade do instrumento para identificar índice de atividade física habitual, a falta de outros estudos referentes à associação da prevalência do BIAFH por meio do questionário Baecke et al. ${ }^{9}$, restringiu a possibilidade de comparação com outras publicações. A utilização do questionário de Beacke et al. ${ }^{9}$, deve-se à facilidade nas respostas e quantificação dos resultados ${ }^{23}$. O escore de atividade ocupacional além de incluir o tipo de ocupação com base no gasto energético, avalia, também, outras atividades físicas laborais (sentar, ficar em pé e andar, carregar carga pesada, sentir-se cansado após a jornada de trabalho, suar no trabalho), e ainda permite a comparação da atividade física no trabalho com pessoas da mesma faixa etária ${ }^{23}$.

A prevalência do BIAFH encontrada foi semelhante à encontrada na literatura, sendo as mulheres mais inativas do que os homens, mesmo após análise ajustada. Porém, apesar da associação do BIAFH com o sexo feminino na maioria dos estudos, iniciativas de sensibilização e de aderência na participação masculina nos programas de saúde pública devem-se tornar mais frequentes.

Os resultados aqui encontrados são importantes para elaboração de programas de intervenção, mostrando limitações e possibilidades de promover atividade física na população estudada. Porém, apesar das associações permitirem reflexões sobre a metodologia das iniciativas para promoção da atividade física, o grande desafio nos programas é fazer com que as mensagens de incentivo e adoção cheguem até as populações de risco, com baixa renda e baixa escolaridade, com desigualdades tão marcantes e com prioridades nas condições básicas de vida ainda não acessíveis.

\section{CONCLUSÃO}

Diante dos resultados apresentados por este estudo, conclui-se que: a prevalência de BIAFH na amostra investigada foi de $51,1 \%$, sendo semelhante aos valores encontrados na literatura; o BIAFH apresentou-se associado com o sexo, com chance maior para as mulheres. É necessário que estudos associem os tipos de atividades físicas (atividades domésticas, lazer, esporte ou ocupação/trabalho) com outras variáveis, o que permitirá o conhecimento das diferentes características da população de acordo com os escores de atividade física específica, indicando as limitações e possibilidades para elaboração de programas de intervenção de atividade física nas populações atendidas pela ESF.

\section{REFERÊNCIAS BIBLIOGRÁFICAS}

1. Bauman A E. Updating the evidence that physical activity is good for health: an epidemiological review 2000-2003. J Sci Med Sport 2004;7(Suppl 1):6-19.

2. Haskell W L, Lee I M, Pate R R, Powell K E, Blair S, Franklin B A, et al. Physical activity and public health: updated recommendation for adults from the American College of Sports Medicine and the American Heart Association. Circulation 2007;116(9):1081-1093.

3. Hallal P C, Dumith S C, Bastos J P, Reichert F F, Siqueira F V, Azevedo M R. Evolução da pesquisa epidemiológica em atividade física no Brasil: revisão Sistemática. Rev Saúde Pública 2007;41(3):453-460.

4. Lessa I, Araújo M J, Magalhães L, Almeida Filho N, Aquino E, Costa M C R. Simultaneidade de fatores de risco cardiovascular modificáveis na população adulta de Salvador (BA), Brasil. Rev Panam Salud Publica 2004;16(2):131-137.

5. Monteiro C A, Conde W L, Matsudo S M, Matsudo V R, Bonseñor I M, Lotufo P A. A descriptive epidemiology of leisure-time physical activity in Brazil, 1996-1997. Rev Panam Salud Publica 2003;14(4):246-254.

6. Hallal P C, Victora C G, Wells J C K, Lima R C. Physical inactivity: prevalence and associated variables in Brazilian adults. Med Sci Sports Exerc 2003;35(11):18941900.

7. Matsudo S M, Matsudo V R, Araújo T, Andrade D, Andrade E, Oliveira L, et al. Nível de atividade física da população do Estado de São Paulo: análise de acordo com o gênero, idade, nível socioeconômico, distribuição geográfica e de conhecimento. Rev Bras Ciên e Mov 2002;10(4):41-50.

8. Matsudo S M, Araújo T, Matsudo V R, Andrade D, Andrade E, Oliveira L, et al. Questionário Internacional de Atividade Física (IPAQ): estudo de validade e reprodutibilidade no Brasil. Rev Bras Ativ Fís e Saúde 2001;6:5-18.

9. Baecke J A, Burema J, Frijters J E. A short questionnaire for the measurement of habitual physical activity in epidemiological studies. Am J Clin Nutr 1982;36(5):936-942.

10. Florindo A A, Latorre MRDO. Validação do questionário de Baecke de avaliação da atividade física habitual em homens adultos. Rev Bras Med Esporte 2003;9(3):121-128. 
11. Pitanga F J, Lessa I. Prevalência e fatores associados ao sedentarismo no lazer em adultos. Cad Saúde Pública 2005;21(3):870-877.

12. Silva M A M, Rivera I R, Ferraz M R M T, Pinheiro A J T, Alves S W S, Moura A A, et al. Prevalência de fatores de risco cardiovascular em crianças e adolescentes da rede de ensino da cidade de Maceió. Arq Bras Cardiol 2005;84(5):387-392.

13. Instituto Brasileiro de Geografia e Estatística. População e Domicílios-Censo 2000 com divisão territorial 2001;Disponível em:<http://www.ibge.bov.br/cidadesat $>$ [2006 jul 14].

14. World Health Organization. Report of a WHO consultation on obesity. Preventing and managing the global epidemic. Geneve: WHO; 1998.

15. Brasil, Ministério da Saúde. Inquérito domiciliar sobre comportamentos de risco e morbidade referida de doenças e agravos não transmissíveis: Brasil, 15 capitais e Distrito Federal, 2002-2003. Rio de Janeiro: INCA; 2004

16. Associação Brasileira de Empresas de Pesquisa - ABEP. Critério de Classificação Econômica Brasil. 2003; Disponível em: <http://www.abep.org> [2006 abr 12].

17. Alvarez R B, Pavan A L. Alturas e comprimentos corporais. In: Petroski EL. Antropometria: técnicas e padronizações. 2. ed. Santa Maria: Pallotti; 2009. p. 31-44.

18. Victora C G, Huttly S R, Fuchs S C, Olinto M T. The role of conceptual frameworks in epidemiological analysis: a hierarchical approach. Int J Epidemiol 1997;26 (1):224-227.

19. Baretta M, Baretta E, Peres K G. Nível de atividade física e fatores associados em adultos no Município de Joaçaba, Santa Catarina, Brasil. Cad Saúde Pública 2007;23(7):1595-1602.

20. Barros M V, Nahas M V. Comportamentos de risco, auto-avaliação do nível de saúde e percepção de estresse entre trabalhadores da indústria. Rev Saúde Pública 2001;35(6):554-563.
21. Dias-da-Costa J S, Hallal P C, Wells J C K, Daltoé T, Fuchs SC, Menezes A M B, et al. Epidemiology of leisuretime physical activity: a population-based study in Southern Brazil. Cad Saúde Pública 2005;21(1):275-282.

22. Coqueiro R S, Nery A A, Cruz Z V, Sá C K C. Fatores associados ao sobrepeso em adultos acompanhados por uma unidade de saúde da família. Rev Bras Cineantropom Desempenho Hum 2008;10(2):149-154.

23. Florindo A A, Latorre M R D O, Jaime P C, Tanaka T, Zerbini C A F. Metodologia para a avaliação da atividade física habitual em homens com 50 anos ou mais. Rev Saúde Pública 2004;38(2):307-314.

\section{Agradecimentos}

Ao Departamento de Saúde Coletiva da Prefeitura Municipal de Guanambi e ao NAFEL/UNEB pelo apoio financeiro.

\section{Endereço para correspondência}

Marcius de Almeida Gomes

Universidade do Estado da Bahia

Campus XII, Departamento de Educação

Linha de Estudo, Pesquisa e Extensão

em Atividade Física

Avenida Universitária Vanessa Cardoso

e Cardoso, Bairro Ipanema, Campus Universitário

Guanambi, (BA), Brasil.

CEP:46430-000

E-mail: marcius_lepeaf@hotmail.com

ou magomes@uneb.br 